\title{
La importancia de las redes sociales en las MiPyMES en tiempos de Covid-19: caso de estudio Orizaba, Veracruz, México
}

The importance of social networks in MSMEs in times of Covid-19: case Study Orizaba, Veracruz México

A importância das redes sociais nas MPMEs em tempos de Covid-19: estudo de caso Orizaba, Veracruz, México

María Edith Quezada Fadanelli

edith_quezada_p40@zongolica.tecnm.mx

Tecnológico Nacional de México, Campus Zongolica - México https://orcid.org/0000-0002-1403-9980

Gabriel Ruiz Contreras

gabriel_ruiz_ige@zongolica.tecnm.mx Tecnológico Nacional de México, Campus Zongolica - México https://orcid.org/0000-0003-0257-0253

Marlene Munguía Martínez marlene_munguia_57@zongolica.tecnm.mx Tecnológico Nacional de México, Campus Zongolica - México https://orcid.org/0000-0002-2586-7634

\section{RESUMEN}

Como causa de la pandemia del COVID-19 el mundo sufrió grandes repercusiones en diferentes ámbitos, los países en vías de desarrollo fueron los más afectados, el caso de México no fue la excepción; diversas empresas se vieron en la necesidad de cerrar definitivamente y otras incursionaron en la era tecnológica. Teniendo como antecedente el contexto anterior surge el interés de realizar un estudio que muestra la importancia de las redes sociales en las Micro, Pequeñas y Medianas Empresas (MiPyMES), siendo un factor importante para poder competir en un mercado global donde las tecnologías de información y comunicaciones (TIC) son una herramienta primordial para la operatividad de una empresa. El objetivo de la investigación es analizar cómo usan las redes sociales las unidades económicas de la región de Orizaba, Veracruz, México. El tipo de investigación es cuantitativa, descriptiva y transversal, se aplicó un cuestionario en línea a 78 empresarios. Los resultados exponen un crecimiento en las ventas de aquellas que tienen de 2 a 3 años utilizando las redes sociales para promocionar su negocio y/o producto, la red más utilizada es Facebook y el principal uso es para la promoción de un producto o servicio.

Palabras clave: MiPyMES, redes sociales, TIC, promoción, Covid-19

\begin{abstract}
As a result of the COVID-19 pandemic, the world suffered great repercussions in different areas, developing countries were the most affected, the case of Mexico was no exception; various companies found it necessary to close definitively and others entered the technological era. Taking the previous context as background, the interest arises to carry out a study that shows the importance of social networks in Micro, Small and Medium Enterprises (MSMEs), being an important factor to be able to compete in a global market where information technologies and communications (ICT) are an essential tool for the operation of a company. The objective of the research is to analyze how the economic units of the Orizaba region, Veracruz, Mexico uses social networks. The type of research is quantitative, descriptive and transversal, an online questionnaire was applied to 78 companies. The results show a growth in sales of those who have been using social networks for 2 to 3 years to promote their business and/or product, the most used network is Facebook and the main use is for the promotion of a product or service.
\end{abstract}

Keywords: MSMEs, social networks, information and communications technology, promotion, Covid-19

\section{RESUMO}

Como causa da pandemia de COVID-19, o mundo sofreu grandes repercussões em diferentes áreas, os países em desenvolvimento foram os mais afetados, o caso do México não foi exceção; várias empresas foram obrigadas a fechar definitivamente e outras entraram na era tecnológica. Tomando o contexto anterior como pano de fundo, surge o 
interesse em realizar um estudo que mostre a importância das redes sociais nas Micro, Pequenas e Médias Empresas (MPMEs), sendo um fator importante para poder competir num mercado global onde as tecnologias de informação e comunicações (TIC) são uma ferramenta essencial para o funcionamento de uma empresa. O objetivo da pesquisa é analisar como as unidades econômicas da região de Orizaba, Veracruz, México, utilizam as redes sociais. O tipo de pesquisa é quantitativa, descritiva e transversal, foi aplicado um questionário online a 78 empresários. Os resultados mostram um crescimento nas vendas de quem utiliza as redes sociais há 2 a 3 anos para divulgar seu negócio e/ou produto, a rede mais utilizada é o Facebook e o principal uso é para a promoção de um produto ou serviço.

Palavras-chave: MPMEs, redes sociais, TIC, promoção, Covid-19

\section{INTRODUCCIÓN}

En la actualidad las MiPyMES enfrentan grandes retos, uno de ellos ha sido la pandemia del COVID-19 con el brote del coronavirus en el año 2019, de acuerdo a la Organización Mundial de la Salud [OMS] (2010), una pandemia acontece cuando una enfermedad se dispersa por todo el mundo. Particularmente, América Latina y el Caribe han tenido que hacer frente al coronavirus en una posición más frágil que el resto del planeta. Hubo una recesión económica, al disminuir las actividades con sus socios comerciales, bajas en precios de productos de primera necesidad, pausa de cadenas globales de valor, caso de los sectores manufactureros que en México y Brasil son los de mayores dimensiones en la zona, el turismo y el área financiera. Los daños debido a la pandemia han sido cuantiosos y han afectado a las actividades económicas de diversas maneras (CEPAL, 2020).

La forma como las empresas realizaban las operaciones antes de la pandemia del COVID-19 tuvo que cambiar abruptamente, sólo aquellas entidades económicas que lograron evolucionar de manera efectiva en el uso de las TIC y el internet permanecieron vigentes. Esta particularidad forzó a que empresas de diferentes tamaños, sectores económicos y regiones geográficas se apropiaron de herramientas tecnológicas para soportar sus actividades cotidianas del negocio, adaptarse a la dinámica del entorno y así poder competir.

Uno de los problemas que aquejan a las MiPyMES es la escasa capacidad para brindar productos o servicios que se diferencien de sus competidores y que sean innovadores, resultado del precario grado tecnológico que poseen, de no tener conocimiento especializado, brechas digitales, acceso a la información, créditos financieros y la contratación de servicios externos (Mendoza y Valenzuela, 2014).

Según datos del Gobierno de México (2020) en la Encuesta Nacional sobre Productividad y Competitividad de las Micro, Pequeñas y Medianas Empresas (ENAPROCE) 2018 indica que existen más de 4.1 millones de empresas clasificadas en los sectores productivos, comercio y servicios privados no financieros; representando las microempresas el $96 \%$ respectivamente.

Por otra parte, la Asociación Mexicana de Internet (AMICIP, 2016) menciona que seis de cada diez empresas mexicanas realizan alguna acción de publicidad o mercadotecnia en internet, de ellas más del $80 \%$ cuenta con un perfil en alguna red social, ocupando el primer lugar Facebook.

El uso de las redes sociales se ha tornado esencial para impulsar a las empresas. Los clientes se interesan por los productos o servicios que ofertan las empresas, surgiendo el marketing digital que cada día está al alza, vislumbrando nuevos segmentos de mercado, que no existirían sin internet (Martínez-Oviedo y Reynoso-Ibarra, 2016).

Sin lugar a duda las redes sociales ayudan a obtener datos importantes para las empresas, ya que proporcionan estadísticos precisos que permiten visualizar el impacto del marketing digital, estar al tanto de los intereses de los clientes potenciales, establecer un canal de comunicación más cercano, y conocer su preferencia por algún producto o servicio. 
Por otra parte, el estudio de las redes sociales en las MiPyMES en tiempos del Covid-19 se realizó en la ciudad de Orizaba, Veracruz, México que de acuerdo con el Comité Estatal de Información Estadística y Geográfica de Veracruz [CEIEG] ( 2019) se encuentra ubicada en la zona centro del mencionado Estado, con una población de 120,844 habitantes, cuenta con un área de $27.97 \mathrm{~km}$ cuadrados; es conocida como la "Ciudad de las Aguas Alegres" y también como "Pluviosilla" y desde el 2015 es considerado "Pueblo Mágico". En esta ciudad ha existido un gran desarrollo industrial, de servicios y comercial, siendo un referente importante para efectuar la investigación.

\section{FUNDAMENTACIÓN TEÓRICA}

La economía mundial vive una dificultad sanitaria y económica donde la inestabilidad de las Micro, Pequeñas y Medianas Empresas es visible. El objetivo del estudio muestra la importancia de las redes sociales en las MiPyMES, considerando un factor de importancia para competir en un mercado global donde la práctica de las redes sociales que seleccionaron las MiPyMES en respuesta a las consecuencias que resultan por los efectos de la pandemia COVID-19 en un lugar.

De acuerdo a Celaya (2008), las redes sociales son espacios virtuales (internet), donde las personas difunden, comparten e intercambian un sin fin de información diversa, desde lo personal, laboral, cultural, académico y profesional, con terceras personas, familiares, conocidos y desconocidos. Por lo cual es un espacio diseñado en un ambiente virtual para simplificar y favorecer la interacción entre personas que se encuentran en diferentes puntos del mundo.

Para Lugo (2012), son una forma de reunir a las personas y empresas que tienen como principal princípio aportar el más innovador instrumento de comunicación que procura y/o requiere, satisfacer las necesidades de la sociedad, estructurada con metas comunes y específicas, la transformación de desarrollo global ha logrado encaminar a las redes sociales como nuevas tecnologías que se involucran en el cambio de la expresión informática del mundo.

Las redes sociales son un impacto en el mundo al momento de la interacción social, a niveles empresariales entre sus actores, configurar los sistemas, contextos sociales, creación de conocimiento e interrelaciones personales. El sistema de redes sociales económicamente muestra el incremento y expansión de una marca o MiPyMES. Por lo cual, la importancia de las redes, como hecho social, cambia la manera en que las personas se interrelacionan con la información.

De acuerdo a Arenas (2013), el ciberespacio social es el que se refiere y se enfoca a atender a las personas, donde es más cómodo mantenerse conectado con la información en todas las áreas y contextos, así como los sucesos que pasan en tiempo real, el espacio que todos tienen el libre albedrío de dar voz y opinión, y beneficio de mantener contacto con las personas, instituciones, organizaciones, etc., es de manera inimaginable que las redes sociales han desencadenado una evolución en la forma de relacionarse y dar a conocer el mundo actual.

Celaya (2008), clasifica las redes sociales de la siguiente forma: 1. Redes profesionales (por ejemplo, LinkedIn, Xing, Viadeo) 2. Redes generalistas (por ejemplo, MySpace, Facebook, Tuenti, Hi5) 3. Redes especializadas (por ejemplo, Ediciona, eBuga, CinemaVIP, 11870), se conoce que existe variedad de redes sociales, estas son las que atienden a un grupo general y se consideran dentro de las que tienen un mayor indicador de consultas, según registros oficiales.

Las redes sociales se encuentran modificando la presencia, intervención y la responsabilidad de los consumidores (Liang y Turban, 2011), con esto, las MiPyMES se han orientado en utilizar las ventajas, la dispersión de la red y sus materiales, apertura un medio de comunicación que concede el flujo de conexión de personas día a día (De Ugarte, 2006). Con mayor frecuencia las personas requieren el uso de formatos online con el propósito de conformar equipos de trabajo e intercambiar mejores prácticas (Kozinets, 2002). Ciertos autores consideran la importancia de las 
redes sociales como una de las herramientas de innovación en la manera de competir de las MiPyMES, y que su investigación integra un excelente instrumento para la recolección de información trascendente (Gálvezy Martín, 2009). Desde que las MiPyMES han sido consideradas como un impulsor para cualquier economía a nivel mundial, se han investigado los factores para analizar los que afectan su auge y desarrollo (Claudino et al., 2017).

En un estudio realizado en Ecuador referente a las redes sociales se encontró que los microempresarios se limitaban a usar el internet para enviar correos y tareas administrativas, a diferencia de las grandes empresas que aprovechan al máximo las redes sociales con campañas publicitarias efectivas. También, se identificó que la red más usada es Facebook, seguida de WhatsApp; por otra parte, se apreció un incremento en el uso de las redes sociales, siendo primordial el trabajo interno en las empresas y replantearse las formas de relacionarse con sus clientes (López et al, 2018).

De forma similar se llevó a cabo una investigación en Colombia donde se proponía al marketing digital como área de oportunidad para la digitalización de las empresas en tiempos de Covid-19, dicho estudio mostró que el $20 \%$ de las pequeñas y medianas empresas estaban en riesgo de cerrar debido a la pandemia y se expusó que el uso del marketing digital es una estrategia importante para la examinar los mercados digitales, con la finalidad de mejorar la economía nacional (Hoyos-Estrada \& Sastoque-Gómez, 2020).

Por otra parte, en un estudio que se realizó en la ciudad de Córdoba, Veracruz, México donde se analizó a 84 microempresarios referente al uso de las redes sociales ante el efecto Covid19 se encontró que el $31 \%$ de ellos disminuyeron sus ingresos, el $24 \%$ comentó la necesidad de incrementar el financiamiento y el $16 \%$ se vió obligado a despedir a sus empleados. También, mencionaron que incrementaron en un $30 \%$ el uso de redes sociales como estrategia ante la pandemia del Covid-19 y las redes más usadas fueron Facebook y WhatsApp para promocionar, dar seguimiento a los clientes, posicionar productos y explorar mercados (Demuner-Flores, 2021). Como se puede apreciar las redes sociales son un soporte fundamental para que los microempresarios puedan subsistir ante la pandemia que se vive actualmente. Las estrategias que han adoptado permiten incursionar en nuevos mercados, donde el uso de las TIC es de vital importancia para poder competir en la nueva normalidad.

Las MiPyMES son el pilar de la estructura económica de México, durante 2019 representaron el $99.8 \%$ de las empresas y participaron con el 67.9\% en el empleo (INEGI, 2019). En el Estado de Veracruz, las MiPyMES ocupan un porcentaje del 99.9\% y también representan el 85.2\% del empleo. De acuerdo al censo empresarial 2017-2018 en Orizaba, Veracruz, México donde se analizaron 2348 MiPyMES mismo que se hizo en colaboración con la Cámara Nacional de Comercio, Servicios y Turismo de esta ciudad, reveló que sólo un $17.25 \%$ hacían uso de página web, redes sociales y mercadotecnia en línea como estrategia de negocio. Estos resultados, mostraron que aún hay un porcentaje bajo de empresarios que utilizan las TIC como estrategia competitiva, lo que muestra la oportunidad para trabajar en esta área (Quezada-Fadanelli, RuizContreras, Velásquez-Cortés y Sánchez-Anastacio, 2018).

Debido a la importancia de las MiPyMES en la economía nacional, así como lo antes mencionado expone el interés por estudiar a las unidades económicas del municipio de Orizaba, Veracruz, México ya que desde antes de la pandemia del Covid-19 se puede observar que hay un porcentaje bajo correspondiente al $17.25 \%$ que usaban las TIC para apoyo a su negocio, evidenciando la necesidad de ampliar su uso a más empresas.

Las redes sociales hacen posible el acercamiento con el cliente y la difusión de información, son una alternativa importante para realizar estrategias de marketing y llevar a cabo la comercialización de productos o servicios (Fonseca, 2014; Palacios, Soto y Merigo, 2015; Pico y 
Coello, 2017; Villagómez y Acosta, 2020) permiten una amplia conexión con más clientes para promoción empresarial (Akdogan y Altuntas, 2015).

La entrada de lo online inició una revolución en la manera en que establecemos las relaciones y las comunicaciones. El marketing digital ha dado una solución a las nuevas tecnologías y a la reciente forma de aprovechar y usar el internet (Castaño \& Jurado, 2016). A través de las redes sociales, las empresas se dan a conocer y obtienen información que mejora las destrezas de sus colaboradores para generar valor en los servicios o productos, así como participar en mercados específicos donde se quiera competir (Camisón y Forés, 2010).

El uso de las redes sociales permite que las MiPyMES estén comunicadas en un mundo globalizado, ya que son una herramienta de la Web 2.0 que es de bajo costo (Rivera, Gutiérrez y Olvera, 2020). El marketing digital ayuda a las empresas a obtener nuevos clientes, interactuar con ellos y a su vez con proveedores, lo que permitirá tener un alcance más amplio (Ainin et al., 2015). Según el reporte anual de The Global State of Digital, en 2020 más del cincuenta por ciento de la población global utiliza las redes sociales, ya sea para la comunicación entre usuarios y para posicionar una empresa (Digital, 2020). En otro informe, se menciona que las redes más usadas en el mundo son Facebook, YouTube y WhatsApp, caso similar en México donde el 69\% de la población usan redes sociales de sus 128.3 millones de habitantes (Digital, 2020a).

\section{PROCEDIMENTOS METODOLÓGICOS}

\subsection{Tipo de investigación}

La investigación utilizó el método cuantitativo para medir el uso de las redes sociales en las MiPyMES, es del tipo descriptivo por qué buscó identificar si el uso de las redes sociales ha incrementado las ventas, se buscó información referente al uso de la red social y transversal porque en un sólo momento del tiempo se recopiló la información.

\subsection{Población}

Se consideró una población de 2348 empresarios de acuerdo al censo empresarial 2017-2018 de la ciudad de Orizaba, Veracruz, México.

\subsection{Muestra}

Se aplicó la fórmula del autor Aguilar-Barojas (2005), tomando un margen de error del 10\% y un nivel de confianza del $90 \%$. Se determinó un total de 66 microempresarios, aunque en la práctica se obtuvieron 78 respuestas.

\subsection{Unidad de Análisis}

Microempresarios de la ciudad de Orizaba, Veracruz, México.

\subsection{Técnicas de recolección de datos}

La técnica utilizada fue la encuesta, la cual se aplicó a los microempresarios del municipio de Orizaba, Veracruz, México.

\subsection{Instrumentos de recolección de datos}

Se utilizó un cuestionario estructurado conformado por 21 ítems. Este instrumento fue adaptado de un estudio referente a las redes sociales (Martínez-Oviedo et al, 2016). Se dividió en tres secciones principales: 
1) Datos Generales: Se trata de recopilar información sobre el dueño o administrador de la empresa, género, edad, nivel de estudios y antigüedad en la empresa.

2) La segunda parte se refiere al tamaño de la empresa, giro, si posee equipo de cómputo, si cuenta con servicio de internet y si cuenta con página web.

3) Por último, se identifica información referente al uso de las redes sociales, qué red es la que usa, tiempo usando la red social, porcentaje en que se incrementaron las ventas, beneficio de la red social, principal uso de la red social, si cuenta con un plan de publicidad, contribución de la red social y cuánto invierte en publicidad digital en la red social.

\subsection{Aplicación del instrumento}

Se utilizó un formulario en línea para facilitar el acceso al mismo, ya que debido a la pandemia del Covid-19 era complicado aplicarlo de forma presencial. El acercamiento a los elementos encuestados fue a través de la vinculación con la Cámara Nacional de Comercio y Turismo de Orizaba, Veracruz, y organismos gubernamentales de la ciudad con quienes se colabora en diversos proyectos. El periodo de aplicación fue en el mes de octubre de 2020.

\subsection{Validación del instrumento}

La validación del instrumento determinó un 0.80 de confiabilidad. Como menciona Oviedo y Campos (2015) un Alfa de Cronbach de .89 a .80 es bueno. Los ítems se clasificaron como: tipo de red utilizada y sus aplicaciones, tiempo de uso de la red social, inversión e impacto en la empresa, entre otros.

\subsection{Método de análisis de datos}

Se empleó la estadística descriptiva, para determinar el nivel en cada variable con la información obtenida de todos los resultados de la encuesta, se procedió a realizar la tabulación de la información en el software Excel, para luego proceder a mostrar los resultados en una gráfica. También se utilizó el software Spss para la determinación del Alfa de Cronbach.

\section{RESULTADOS Y DISCUSIÓN}

Se presentan los resultados más relevantes del estudio, referente al giro predominante de los microempresarios que usan las redes sociales es $55 \%$ pertenece al sector servicios, un $24 \%$ a la comercialización de productos y un $20 \%$ producción y comercialización, como se puede apreciar en la figura 1.

\section{Figura 1}

Giro del negocio

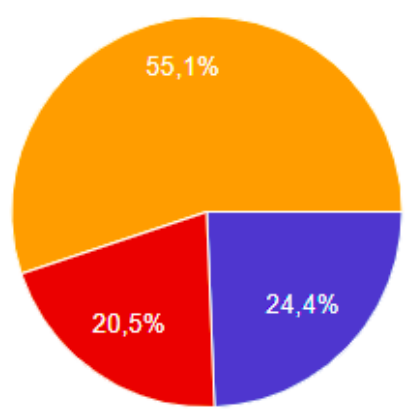

Producción y comercialización

Comercialización

Servicio 
Referente a la pregunta de la antigüedad de la empresa el $55 \%$ de los encuestados afirma tener un tiempo menor a cinco años, el $21 \%$ de cinco a 10 años, el $15 \%$ de once a veinte años y solamente un $7 \%$ tiene más de veinte años en operación (figura 2).

\section{Figura 2}

Antigüedad de la empresa

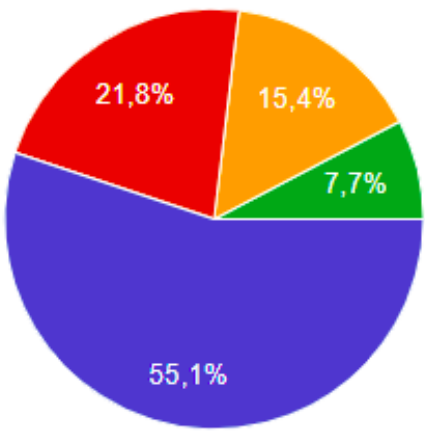

Las MiPyMES suelen presentar mayor variabilidad en cuanto a sus ingresos, personal ocupado, ubicación, antigüedad, cierres y aperturas, entre otros aspectos. Aunado a este comportamiento natural, la pandemia por el brote del COVID-19 llevó a los negocios a pausar sus actividades o incluso a cerrar de manera definitiva (INEGI, 2020).

En cuanto a la pregunta si contaban con internet en su empresa el 91\% respondió que sí y sólo un $9 \%$ no contaban con el servicio, como lo muestra la figura 3. Estos resultados muestran que al contar con el servicio de internet los empresarios cuentan con áreas de oportunidad para sus negocios.

\section{Figura 3}

Empresas con servicio de internet

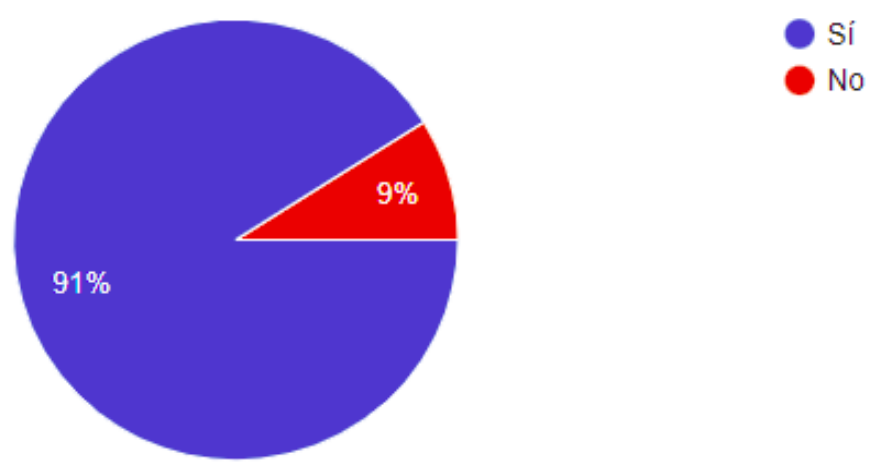

Por otra parte, los resultados reportan que la red social que más utilizan los empresarios encuestados es Facebook con un $65 \%$ y le sigue WhatsApp con un $21 \%$, mostrando la tendencia global que hay del uso de estas redes, como se puede apreciar en la figura 4.

\section{Figura 4}

Red social más utilizada 


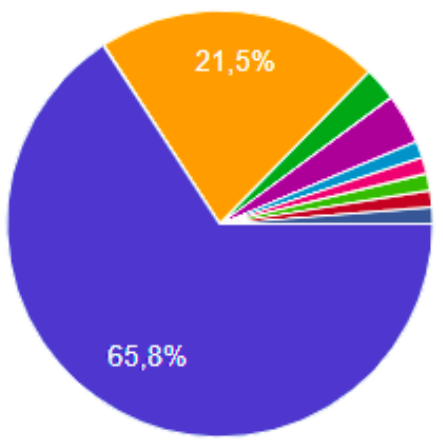

Facebook

Twitter

WhatsApp

Instagram

No utilizo

Google

Página web

Personalmente

De acuerdo al reporte anual de The Global State of Digital (2020) donde se informa que la red más utilizada en el mundo y de igual manera en México es Facebook, este mismo caso se presenta en el municipio de Orizaba, Veracruz donde se puede observar que los empresarios también prefieren usar dicha red social, ya que ocupa el primer lugar en uso.

El tiempo que llevan los empresarios utilizando una red social el 56\% tiene de 2 a 3 años, el $17 \%$ al menos un año, el 15\% de 1 a 2 años y el 10\% menor de 6 meses. Estos resultados reflejan que los encuestados si hacen uso de las redes sociales, en la figura 5 se muestran los porcentajes.

\section{Figura 5}

Tiempo usando una red social

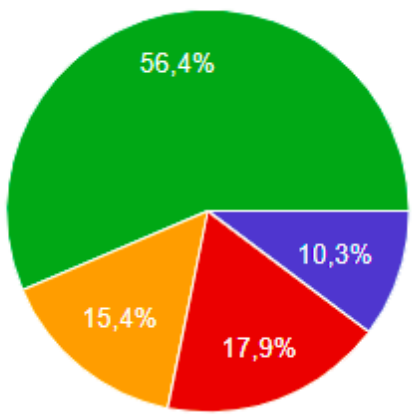

Otro resultado interesante en los encuestados, es la relación del incremento de las ventas en relación al tiempo usando una red social, estos muestran que los que han utilizado de 2 a 3 años sus ventas han aumentado en un $40 \%$ (figura 6).

\section{Figura 6}

Porcentaje de incremento en las ventas con relación al tiempo usando la red social

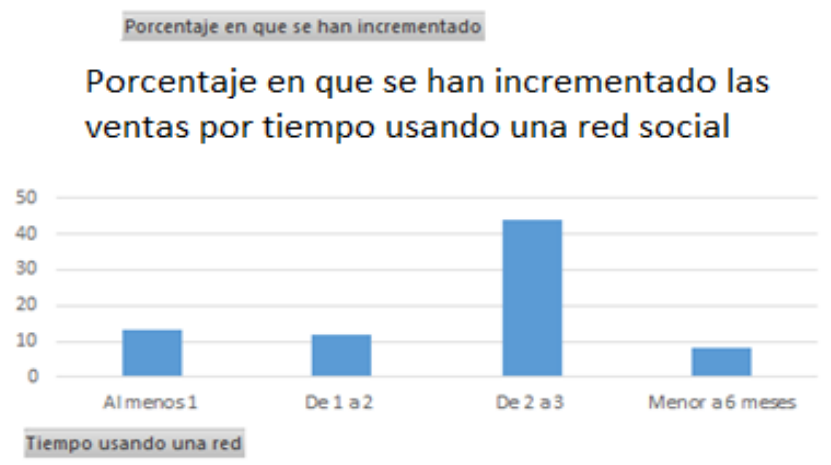


Respecto a los principales beneficios del uso de la red social el $57 \%$ de los encuestados comentan que fue para dar a conocer el producto o servicio, el $25 \%$ notó un incremento en sus ventas y el $16 \%$ para posicionamiento de la empresa (figura 7).

\section{Figura 7}

Principales beneficios del uso de la Red Social

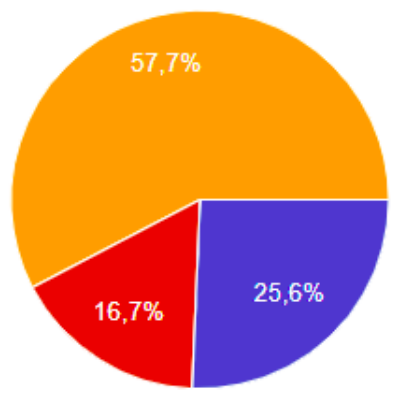

Incremento en las ventas

Posicionamiento de la empresa

Dar a conocer el producto/servicio

Tomando en cuenta la percepción de los encuestados se les preguntó la contribución del uso de redes sociales en su empresa, el $76 \%$ destacó el acercamiento con el cliente, el $11 \%$ el incremento en la productividad y un $9 \%$ el conocimiento del mercado (figura 8 ).

\section{Figura 8}

Contribución del uso de las redes sociales en la empresa

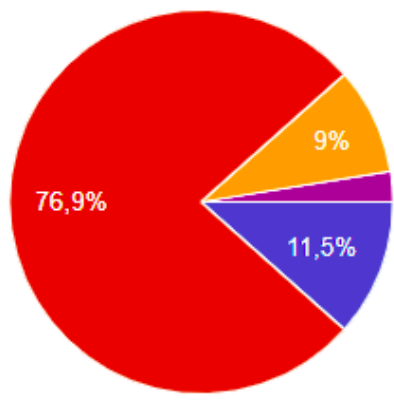

Incremento en la productividad

Acercamiento con el cliente

Conocimiento del mercado

Mejora del producto

Optimización del tiempo

Por otro lado, el 52\% de los encuestaron manifestaron que no cuentan con un plan de publicidad o promoción, el 28\% tienen parcialmente un plan y sólo el $19 \%$ cuenta con un plan; los resultados reflejan un área de oportunidad para realizar acciones que los acerquen a los clientes y se incrementen sus ventas (figura 9).

\section{Figura 9}

Plan de publicidad o promoción

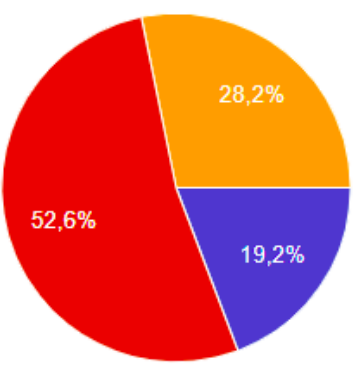


De acuerdo a un estudio realizado a 5 mil unidades económicas por una empresa especializada en soluciones tecnológicas en el 2015 en Estados Unidos, el 70 por cierto de los encuestados planea aumentar la publicidad en redes sociales, plataformas como: Facebook, Twitter, Instagram y Snapchat (Gómez, 2015).

Como se puede observar en este estudio menos del $20 \%$ de los empresarios cuentan con un plan publicitario en Orizaba, Veracruz, por lo que se sugiere realizar acciones que permitan impulsar estrategias publicitarias en esta área; si bien hablamos de nuestro país vecino por la parte norte que sus condiciones versan a las de México, sin embargo, es importante considerar los beneficios que puede aportar un plan publicitario para una empresa.

Por último, se presenta la opinión de los micro empresarios referente a si la red social ha contribuido al fortalecimiento de su negocio, el $66 \%$ comenta que sí y el $23 \%$ dicen que en poca medida. Como se puede apreciar, un porcentaje mayor son los que consideran que las redes sociales fortalecen su negocio (figura 10).

\section{Figura 10}

Percepción, si la red social ha contribuido al fortalecimiento del negocio

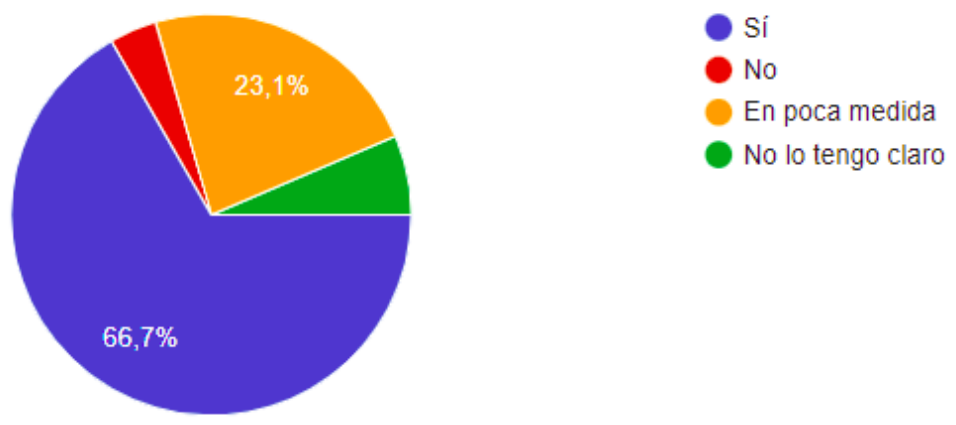

La mayoría de los empresarios reconoce que las redes sociales representan una excelente oportunidad para empoderar su negocio, por otra parte, las redes sociales ofrecen herramientas estadísticas que permiten la segmentación de mercado, conocer las preferencias de los clientes, contenido demográfico y los gustos de cada uno de los consumidores (Valdés, 2015).

\section{CONCLUSIÓN}

La Organización Internacional del Trabajo (2020) menciona que todas las empresas, independientemente de su tamaño, se ven afectadas por la pandemia del Covid-19, sobre todo aquellas correspondientes al sector terciario. Algunas de las afectaciones que tuvieron fue la disminución de sus ingresos, pérdida de empleos, etc. Sin embargo, las más afectadas son las MiPyMES que muchas de ellas se vieron comprometidas para continuar con sus operaciones.

Se puede observar que los empresarios de Orizaba, Veracruz, México tienen herramientas que pueden ser de utilidad, un porcentaje alto de ellos cuentan con internet en sus negocios, si hacen uso de las TIC adecuadamente tendrían un beneficio potencial en sus actividades.

Las redes sociales cada vez se extiende más su uso, sin embargo, no todos los encuestados manifiestan usar el amplio potencial que brindan, es cierto que la pandemia aceleró el uso de ellas, pero se requiere de planes de marketing personalizados que hagan posible la competencia a nivel global, así como hacer frente a la crisis económica que se vive actualmente a raíz del Covid-19. 
Por otra parte, es bien sabido que la pandemia del coronavirus aumentó de manera potencial las actividades en línea tanto económicas como sociales, posicionando al teletrabajo y a la digitalización como tareas primordiales en todos los ámbitos. Se considera que las organizaciones que tengan mayor desarrollo tecnológico tendrán más ventajas en comparación con las que estén atrasadas, específicamente las MiPyMES (CEPAL, 2020).

Se considera necesario profundizar más en la teoria para identificar las estrategias que han dado resultado para fortalecer a las MiPyMES en lo referente al uso del marketing digital, de esta manera podrán resistir el embate de la pandemia. Por otra parte, queda la oportunidad para usar la estadística multivariada que permita encontrar relaciones entre las variables que intervienen.

Finalmente, para trabajos futuros se recomienda ampliar el número de la muestra para tener resultados más precisos estadísticamente que brinden un panorama más certero del contexto de las MiPyMES de la región de estudio.

\section{REFERENCIAS}

Ainin, S., Parveen, F., Moghavvemi, S., Jaafar, N. I. \& Shuib, N. L. M. (2015). Factors influencing the use of social media by SMEs and its performance outcomes. Industrial Management Data Systems, 115(3), 570-588. https://doi.org/10.1108/IMDS-07-2014-0205

Akdogan, M. \& Altuntas, B. (2015). Covert Marketing Strategy and Techniques. Procedia-Social and Behavioral Sciences, 207, 135-148. https://doi.org/10.1016/j.sbspro.2015.10.162

Aldape, N. K. A., Abrego, A. D. \& Medina, Q. J. M. (2016). Análisis de la percepción de uso de las redes sociales como herramienta de marketing en las MiPYMES de Tamaulipas. RISTI, No. 18(6), 49-65. http://dx.doi.org/10.17013/risti.18.49-65

AMIPCI. (Abril de 2016). Asociación Mexicana de Internet. Marketing digital y redes sociales Obtenido de http://www.amipci.org.mx/es/

Arenas, J. (2013). Plan social media y community manager. Madrid. Editorial UOC.

Camisón, C. \& Forés, B. (2010). Knowledge absorptive capacity: New insights for its conceptualization and measurement. Journal of Business Research, 63, 707-715.

Castaño, J. J., \& Jurado, S. (2016). Marketing digital (Comercio electrónico). Madrid: Editex.

CEIEG. (09 de Mayo de 2019). Comité Estatal de Información Estadística y Geográfica de Veracruz, Cuadernillos Municipales. Obtenido de http://www.ceieg.veracruz.gob.mx

Celaya, J. (2008). La Empresa en la WEB 2.0. Editorial Grupo Planeta, España.

CEPAL. (Comisión Económica para América Latina y el Caribe). (2020). América Latina y el Caribe ante la pandemia del COVID-19. Efectos económicos y sociales. Informe especial COVIT-19, No. 1. Editorial: CEPAL, 3 de abril. https://www.cepal.org/es/publicaciones/45337-america-latina-caribe-la-pandemia-covid-19-efectoseconomicos-sociales

Claudino, T., Dos Santos, S., Cabral, A. y Pessoa, M. (2017). Fostering and Limiting Factors of Innovation in Micro and Small Enterprises. RAI. Revista de Administração e Inovação, 14(2), 130-139. https://doi. org/10.1016/j.rai.2017.03.007

Demuner Flores, M. R. (2021). Uso de redes sociales en microempresas ante efectos COVID-19. Revista de Comunicación de la SEECI, 54, 97-118. https://doi.org/10.15198/seeci.2021.54.e660

Díaz, B. J. L., Armas, T. V., Morales, C. J. C. \& Ortigoza, H. A. (2020). Estudio de la mezcla de promoción en las PYMES Poblanas. Revista Relayn Micro y pequeña empresa en Latinoamérica, 4(2), 102-112. http://iquatroeditores.com/revista/index.php/relayn/article/view/92

Digital. (2020). Global Digital Overview v01. https://www.slideshare.net/DataReportal/digital-2020-global-digitaloverview-january-2020-v01-226017535

Digital. (2020a). Digital 2020: México. https://datareportal.com/reports/digital-2020-mexico

Fonseca, A. (2014). Marketing Digital en redes sociales: Lo imprescindible en Marketing Online, Calendar, Palma Área, España.

Gobierno de México (2020). INEGI presenta resultados de la Encuesta Nacional sobre Productividad y Competitividad de las MIPYMES (ENAPROCE) 2018. [fecha de Consulta 10 de Enero de 2022]Recuperado de: 
www.gob.mx/se/articulos/inegi-presenta-resultados-de-la-encuesta-nacional-sobre-productividad-y-competitividadde-las-mipymes-enaproce-2018

Gómez, J. (26 de 01 de 2015). Merca2.0. Obtenido de http://www.merca2.0.com/casi-5-millones-de-empresas-existenen-mexico-inegi/

Hoyos, A., \& Lasso, M. (2017). Pymes como modelo económico en la creación de estrategias de comunicación.

Hoyos-Estrada, S., \& Sastoque-Gómez, J. (2020). Marketing Digital como oportunidad de digitalización de las PY-MES en Colombia en tiempo del Covid - 19. Revista Científica Anfibios, 3(1), 39-46. https://doi.org/10.37979/afb.2020v3n1.60

Ibarra, C. M. A., González, T. L. A. \& Demuner, F. M. R. (2017). Competitividad empresarial de las pequeñas y medianas empresas manufactureras de Baja California. Estudios Fronterizos, 18(35) enero-abril, 107-130. https://doi.org/10.21670/ref.2017.35.a06

INEGI. (2019). Censos Económicos 2019. Micro, pequeña, mediana y gran empresa: estratificación de los establecimientos. Instituto Nacional de Estadística y Geografía. México: INEGI, c2020. https://www.inegi.org.mx/programas/ce/2019/

INEGI. (2020). Resultados de la segunda edición del ECovid-IE. Instituto Nacional de Estadística y Geografía. México: INEGI https://inegi.org.mx/contenidos/saladeprensa/boletines/2020/OtrTemEcon/ECOVID-IE_DEMOGNEG.pdf

López, Oscar et al. Estrategias de marketing digital por medio de redes sociales en el contexto de las PYMES del Ecuador. CienciAmérica, [S.1.], v. 7, n. 2, p. 39-56, jul. 2018. ISSN 1390-9592. Disponible en:

<http://cienciamerica.uti.edu.ec/openjournal/index.php/uti/article/view/167> doi: http://dx.doi.org/10.33210/ca.v7i2.167.

Lugo, A (2012). Los Partidos Politicos en Mexico Y La Sucesion Presidencial del Año 2012. México: Palibrio

Martínez-Oviedo, A. F., \& Reynoso-Ibarra, O. Y. (2016). Las redes sociales como impulsor en el crecimientode los emprendedores y las MiPyMes. Revista de Negocios y PyMES Vol 2 Número 5, 34-44.

Mendoza, J., \& Valenzuela, A. (2014). Aprendizaje, innovación y gestión tecnológica en la pequeña empresa. Un estudio de las industrias metalmecánica y de tecnologías de información en Sonora. Contaduría y Administración, 59(4).

Organización Mundial de la Salud [OMS] (2010). ¿Qué es una pandemia?. Autor. Recuperado de: https://www.who.int/csr/disease/swineflu/frequently_asked_questions/pandemic/es/

Oviedo, H. C., \& Campos, A. (2015). Metodología de la investigación y lectura crítica de estudios. Obtenido de http://www.redalyc.org/pdf/806/80634409.pdf

Palacios, M. D., Ponce, A. J., Villamrin, V. W. \& Palma, A. A. M. (2020). Las redes sociales y su influencia como estrategia de marketing en las PYMES de Manabí - Ecuador. Revista Observatorio de la Economía Latinoamericana, abril, 1-23. https://www.eumed.net/rev/oel/2020/04/redes-sociales-marketing.pdf

Palacios, M. D., Soto, A. P. \& Merigo, J. M. (2015). Analyzing the effects of technological, organizational and competition factors on Web knowledge exchange in SMEs. Telematics and Informatics, 32(1), 23-32. http://dx.doi.org/10.1016/j.tele.2014.08.003

Pico, V. L. M. \& Coello, Y. R. R. (2017). Relación entre el ciclo de vida de las Pymes en redes sociales y el emprendimiento en la ciudad de Guayaquil. INNOVA Research Journal, 2(5), 125-136.

Quezada-Fadanelli, M. E., Ruiz-Contreras, G., Velásquez Cortés, C., \& Sánchez-Anastacio, I. (2018). Censo de MIPYMES de Orizaba , Veracruz como estrategia para detectar áreas de oportunidad que permitan su fortalecimiento. Micro y pequeña empresa en Latinoamérica, 2(2), 40-49. Obtenido de https://iquatroeditores.com/revista/index.php/relayn/article/view/41/37

Real, P. I, Leyva, C. A. B. \& Heredia, B. J. A. (2018). Uso e impacto de las redes sociales en las estrategias de marketing de las PyME's. Revista De Investigación Académica Sin Frontera, 7(19), julio-diciembre, 1-24. https://revistainvestigacionacademicasinfrontera.unison.mx/index.php/RDIASF/article/view/47

Rivera, T. I., Gutiérrez, A. J. \& Olvera, L. M. D. (2020). Websites and Social Networks. A Study of Healthcare SMEs in Andalusia. In: Rocha Á., Ferrás, C., Montenegro, M. C. \& Medina, G. V. (eds) Information Technology and Systems. ICITS 2020. Advances in Intelligent Systems and Computing, vol 1137. Springer, Cham.

Valdéz, A. (2015). Redes Sociales:Impacto en los negocios por Internet. Obtenido de http://wasanga.com//anavaldes/redes-sociales

Villagómez, M. A. R. \& Acosta, G. E. (2020). Uso de redes sociales digitales como estrategia de mercadotecnia en pymes pirotécnicas de Tultepec en el Estado de México. Revista de Comunicación de la SEECI, (52), 73-93. doi: http://doi.org/10.15198/seeci.2020.52.73-93 